Third Day: Wed., Nov. 9

Morning Session. Short-Range Forecast IV (Numerical modeling research)

Numerical Computation of Wind Tides on Lake Erie

\author{
G. W. Platzman
}

The Ekman equations for the transient state of a large, enclosed body of water are derived in an approximate two-dimensional form. These equations are applied to the numerical computation of wind-induced tides on Lake Erie. Several observed cases of large-amplitude tides are computed from hourly wind data, and the results are compared with concurrent records of lake-level observations.

\title{
A Numerical Model for Thermal Convection in the Atmosphere
}

\section{J. G. Charney and Y. Ogura}

The numerical experiments to be described have the goal of studying the dynamical behavior of various forms of thermal convection in a conditionally unstable atmosphere, such as cumulus clouds, tornadoes, squall lines and hurricanes. The model consists of equations of motion, the continuity equation of mass (in which change of density with time is ignored), and the conservation laws of equivalent potential temperature and of water vapor. The hydrostatic relation is not used. The formulation is such that acoustic motion is suppressed and only gravity-inertia motion can occur. The results of numerical integrations will be presented.

\section{A Numerical Model of the Sea Breeze}

\section{A. Estoque}

A primitive equation model is formulated and integrated numerically to study the sea breeze. The formulation is an extension of an atmospheric boundary layer model previously developed for a homogeneous terrain. External gravity wave type solutions are suppressed by a modelling assumption involving the equation of continuity. The results of integrations showing the formation, intensification, and decay of the sea breeze circulation will be presented.

\section{Basic Mesometeorological Research \\ and Its Potential Values in a Short-Range Forecast}

T. FUJiTA

With the use of data collected by mesoscale meteorological networks, the vertical and horizontal extents of various mesoscale disturbances were investigated and classified. 